\title{
La Voz del Silencio: Evaluación Cualitativa de Prácticas de Bullying en Personas con Discapacidad Intelectual ${ }^{1}$ \\ The Voice of Silence: Qualitative Evaluation of Bullying Practices in PEOPLE WITH INTELLECTUAL DISABILITY
}

\author{
María Luisa Belmonte ALMAGRO² \\ Abraham Bernárdez GÓMEZ3 \\ Ana Belén Mirete RUIZ ${ }^{4}$
}

\begin{abstract}
RESUMEN: En los múltiples movimientos académicos acaecidos en pos de la inclusión del alumnado, las instituciones universitarias están abriendo sus títulos a colectivos tradicionalmente olvidados en el ámbito de la educación superior, como las personas con discapacidad intelectual. El acoso escolar es una realidad que puede afectar a todo el alumnado, siendo más complejo conforme avanza la edad de los estudiantes. El objetivo de este trabajo radica en recabar información acerca de la percepción de las personas con discapacidad intelectual sobre el bullying y reconocer las diferentes manifestaciones de acoso a personas con discapacidad intelectual. A través de una investigación biográfico-narrativa y bajo un diseño evaluativo de carácter mixto, se ha contado con la participación de 17 estudiantes que cursan el Título Universitario de Estudios Propios Todos Somos Campus para fomentar la inserción socio-laboral del colectivo de jóvenes con discapacidad intelectual. A través de un análisis cualitativo de los datos, los resultados muestran que existe una situación de acoso, tanto físico como psicológico, basada en críticas y percepciones negativas acerca de la discapacidad, lo cual les hace más sensibles a la condición de diferentes. Igualmente, se refuerza la imagen de autoridad como eje de las acciones contra el bullying, destacando el papel de la mediación en la exposición de soluciones. Se concluye que las relaciones establecidas entre las manifestaciones del acoso en el ámbito académico y sus posibles soluciones no se presentan de forma aislada, sino que se muestran como una red que facilita respuesta a dicha problemática.
\end{abstract}

PALABRAS-CLAVE: Bullying. Discapacidad intelectual. Educación Superior.

ABSTRACT: In the multiple academic movements for the inclusion of students, university institutions are opening their undergraduate degrees to groups traditionally forgotten in the field of Higher Education, such as people with intellectual disabilities. Bullying is a reality that can affect all students, being more complex as the age of the students advances. The objective of this work is to gather information about the perception of people with intellectual disabilities about bullying and, thus, recognize the different manifestations of harassment to people with intellectual disabilities. Through a biographical-narrative research and a mixed evaluative design, we counted on the participation of 17 students who are attending the Titulo Universitario de Estudios Propios Todos Somos Campus - University Degree of Own Studies We are all Campus - to promote the social and labor insertion of the collective of young people with intellectual disabilities. Through a qualitative data analysis, the results showed that there is a situation of harassment, both physical and psychological, based on criticism and negative perceptions about the disability, which make it more sensitive to the status of different. In the same way, it reinforces the image of authority as an anti-bullying action, highlighting the role of mediation in the exposition of solutions. It is concluded that the relations established between the manifestations of bullying in the academic sphere and their possible solutions are not presented in isolation, but they are shown as a network that facilitates the answer to this problem.

KEYWORDS: Bullying. Intellectual disability. Higher Education.

\footnotetext{
${ }^{1}$ https://doi.org/10.1590/1980-54702021v27e0027

${ }^{2}$ Doctorado em Investigación e Innovación en Educación Infantil y Educación Primaria. Departamento de Métodos de Investigación y Diagnóstico en Educación. Universidad de Murcia. Murcia/España. E-mail: marialuisa.belmonte@um.es. ORCID: https:// orcid.org/0000-0002-1475-3690

${ }^{3}$ Máster en Procesos de Formación. Departamento de Didáctica y Organización Escolar. Universidad de Murcia. Murcia/España. E-mail: abraham.bernardez@um.es. ORCID: https://orcid.org/0000-0003-1862-5554

${ }^{4}$ Doctorado em Psicología. Departamento de Métodos de Investigación y Diagnóstico en Educación. Universidad de Murcia. Murcia/Espańa. E-mail: anabelen.mirete@um.es. ORCID: https://orcid.org/0000-0001-6011-8826
} 


\section{INTRODUCCIÓN}

En estos últimos años, las instituciones universitarias se están viendo inmersas dentro de un significativo avance en materia de inclusión educativa (Melero et al., 2019), dado que, directamente proporcional al avance del tiempo, ha proliferado en dicho contexto la diversidad entre el alumnado, junto con la participación e incorporación de colectivos que tradicionalmente se encontraban al margen de la educación superior (Thomas, 2016; Veitch et al., 2018).

Las investigaciones centradas en alumnos con discapacidad intelectual (Díez et al., 2015) evidencian que, con el paso del tiempo, el número de estudiantes de dichas características y los estudios sobre la respuesta de la educación superior a esta nueva situación (Arnaiz, 2019), no cesan de aumentar. Pese a ello, la presencia de dicho alumnado en las instituciones universitarias es preocupantemente reducida (Cerrillo et al., 2013) y la condición de discriminación continua que restringe las actividades de la vida diaria de las personas con discapacidad intelectual continúa siendo un hecho irrefutable, ya sea por tradición, por falta de información (Belmonte \& García-Sanz, 2013).

El peligro de esta desigualdad sufrida por las personas con discapacidad radica en que este germen de inferioridad tiende a propagarse transversalmente a todas las áreas de sus vidas, derivando todo ello en autoevaluaciones negativas, sentimientos de impotencia y frustración (Jahoda \& Markova, 2004). Este horizonte desafiante (Lipka et al., 2019), al que debe hacer frente este colectivo, es una constante desigualdad por motivos de discapacidad (Heikkilä et al., 2020).

En este sentido, dado que la inclusión en la educación superior de personas con discapacidad intelectual contribuye a construir una mejor Universidad (Cerrillo et al., 2013), no cabe duda de que el aprendizaje en las aulas universitarias debe ser inclusivo (Hardy $\&$ Woodcock, 2015), al ser éste un sinónimo de calidad (Gairín \& Suárez, 2014). Pero, la cuestión es, ¿cómo son las relaciones de inclusión?, y más concretamente, ¿cómo abordan estas relaciones las personas con discapacidad intelectual?

Es un error común pensar que la intimidación o el acoso únicamente se desarrolla en etapas educativas inferiores. Recabar esfuerzos para hacer frente al fenómeno del acoso escolar es crucial para lograr una escuela inclusiva. Aunque este reto lamentablemente tenga tintes utópicos, dentro de dicho contexto, todo tipo de alumnado podría desarrollar de forma exitosa sus capacidades sociales y de aprendizaje.

La falta de empatía, altruismo y aceptación de las diferencias, van ligadas a conductas hostiles y agresivas, que son susceptibles de derivar en acoso psicológico (Hernández et al., 2010). Identificar este acoso como un modo de violencia es el primer paso para comprender la diferencia como elemento de exclusión social (Goicoechea \& Biedma, 2017). Así pues, el acoso, tanto en contextos educativos como laborales, es un problema que afecta a todas las etapas de la vida y colectivos, pero especialmente a las personas con discapacidad intelectual (Strand et al., 2004). Aunque, a medida que aumenta la edad, la causa de dicho acoso tiende a complejizarse y adoptar incluso más matices (Adams \& Lawrence, 2011).

El alumnado con discapacidad es más vulnerable a este tipo de violencia, considerándose incluso este hecho, un factor de riesgo (Rose et al., 2011). Además, los perjuicios sufridos 
por este colectivo están equiparados a los de cualquier alumno sin discapacidad (Zeedyk et al., 2014). Las consecuencias fundamentales de este acoso suelen ser la baja autoestima y pérdida de confianza y seguridad, junto con el aislamiento, la soledad y la impotencia.

Pese a todo ello, los estudios sobre bullying y alumnado con discapacidad (Houchins et al., 2016; Vessey \& O’Neill, 2011; Wei et al., 2016), son menores que los del alumnado con desarrollo típico (Tipton-Fisler et al., 2018).

Varios son los países que han puesto en marcha, en la última década, diferentes acciones para la mejora del proceso de inclusión de las personas con discapacidad en las instituciones de educación superior (Riddell \& Weedon, 2014). Desde la Universidad de Murcia (Espańa), y más concretamente dentro del Título Universitario de Estudios Propios Todos Somos Campus (Belmonte et al., 2020), se viene fomentando la inserción socio-laboral del colectivo de jóvenes con discapacidad intelectual, dentro de un contexto universitario de plena inclusión. Con ello, se pretende provocar un cambio de mentalidad en la comunidad universitaria en relación al esfuerzo por la inclusión de este colectivo.

Aunque no es objeto de este artículo desarrollar una presentación detallada de las características del citado título, conviene, en aras de la claridad expositiva, apuntar, muy brevemente, en qué consiste. Dicho programa, cofinanciado por el Fondo Social Europeo y la Iniciativa Empleo Juvenil a través de la convocatoria de ayudas de Fundación ONCE, permite que jóvenes con discapacidad intelectual se formen en la Universidad y se preparen para conseguir un empleo digno. Además, supone para estos jóvenes una formación para la mejora de su autonomía e inclusión socio-laboral, así como también el fomento del desarrollo de la motivación por el aprendizaje y desempeño responsable de tareas. Para la Universidad de Murcia, concretamente, supone establecer un sistema de formación inclusivo y de normalización en el entorno de la comunidad universitaria (Belmonte et al., 2020).

El objetivo de este estudio es recabar información acerca de la percepción de las personas con discapacidad intelectual del Título Universitario de Estudios Propios Todos Somos Campus, sobre el bullying, para reconocer así las diferentes manifestaciones del acoso a personas con discapacidad intelectual. Concretamente analizando las manifestaciones que han presenciado o sufrido y las posibles soluciones que ellos consideran más relevantes para erradicar esta problemática.

\section{Diseño METOdológico}

A continuación, se exponen los métodos utilizados para la investigación. Para el cumplimiento del propósito de este estudio se utilizó un diseño evaluativo (García-Sanz, 2012) dentro de los modelos mixtos (Pereira, 2011), siguiendo un proceso metodológico de investigación cualitativa (Bisquerra, 2014) biográfica-narrativa (Morińa, 2017), las cuales "no solo hablan de los sujetos individuales, sino que ponen de manifiesto los contextos sociales y culturales en los que estas se han ido construyendo" (Flores et al., 2010, p. 121), y que son de interés, puesto que permiten acceder a las experiencias escolares y sociales (Mañas et al., 2020). 


\subsection{Participantes y procedimiento}

La muestra de esta investigación fue la totalidad de los integrantes del II Curso Todos Somos Campus, durante el curso 2018/19, de formación para el empleo y la inclusión universitaria de jóvenes con discapacidad intelectual. La estrategia de muestreo fue no probabilística por conveniencia, empleando este tipo de muestreo a fin de garantizar las características de los sujetos coinciden con las de la población objeto de estudio (McMillan \& Schumacher, 2005). Así, como característica común, se tomó a estos 17 jóvenes de 18 a 30 años, por presentar una discapacidad intelectual reconocida oficialmente por el Instituto Murciano de Acción Social (IMAS) igual o superior al 33\%. Estos estudiantes poseen capacidad de desplazamiento autónomo y habilidades académicas básicas de competencias mínimas en lectoescritura y cálculo.

El procedimiento seguido ha pasado por tres fases, una inicial en la que se llevaron a cabo los preparativos necesarios para la investigación. En la fase de desarrollo se mostró a los alumnos la representación, en tamaño poster, de lo que sería una estudiante cualquiera, y se les explicó que la chica tenía problemas en el centro educativo, con sus compañeros. Que cada día se le hacía más difícil ir a clase. A continuación, debían ponerse en la piel de aquella chica, y en papel de color rojo, responder las cuestiones abiertas sobre manifestaciones de bullying que ellos habían vivido y que pensaban que podría estar sufriendo la protagonista. Después de dicha reflexión, de manera individual y paulatina, debían dibujar en la representación gráfica de la chica acosada, diferentes moratones o cicatrices, según la manifestación de bullying que aportaran. Una herida por cada insulto, una herida por cada acto de violencia. Una vez que todo el alumnado con discapacidad intelectual hubiera aportado su manifestación del bullying y la hubiera plasmado en forma de agravio, en el póster, se procede a evaluar los dańos sufridos por la chica, tratando de dar respuesta a un interrogante: ¿crees que es violencia?. Seguidamente, los estudiantes con discapacidad deben proponer, en láminas de color verde, posibles soluciones para solventar cada uno de los casos de acoso presentados.

Por último, al término del coloquio acerca de lo sucedido, se debate sobre del daño que se le puede ocasionar a una persona. Desde fuera, a veces, no somos conscientes del daño acaecido a alguien, pero siempre tiene repercusiones. En conclusión, sobre cómo, pese a que se busquen (y encuentren) soluciones, siempre queda la cicatriz y nunca más vuelve a ser la misma.

\subsection{TÉCNICAS DE RECOGIDA DE INFORMACIÓN Y ANÁLISIS DE DATOS}

La participación de las personas con discapacidad intelectual en los estudios de investigación supone aun un reto, resultando así muy necesario el desarrollar experiencias donde sus voces adquieran una mayor relevancia (González Luna, 2013). Es por ello que, en esta investigación, para conocer la percepción de las personas con discapacidad intelectual sobre las situaciones de acoso vividas y las posibles soluciones, se utilizó un cuestionario mixto, para mayor comprensión acerca del objeto de estudio (Onghena et al., 2019), compuesto tanto por preguntas sociodemográficas, relativas al género y edad del alumno, junto con ítems cerrados (analizados en un estudio previo), y también cuestiones abiertas. La opción de "respuesta abierta libera aquellos elementos cognitivos de carácter latente en las opiniones de los informantes" (Gálvez, 2012, p.72). 
El conjunto de datos se ha analizado de forma inductiva (Newman, 2006) mediante técnica de análisis de contenido (Miles et al., 2014) sujeta a la teoría fundamentada (Alarcón, et al., 2017; Strauss \& Corbin, 1998), considerada como muy apropiada cuando se pretende averiguar cómo las personas interpretan su realidad (Suddaby, 2006). Para realizar esta acción, se ha empleado un programa para el análisis cualitativo asistido por ordenador, CAQDAS (por sus siglas en inglés), en este caso, el ATLAS.ti V8.

Para realizar este tipo de análisis hay que tener en cuenta la sistematicidad en la codificación (San Martín, 2014), por ello, para tal fin, se ha llevado a cabo una primera codificación abierta, con el fin de identificar los distintos conceptos subyacentes; una segunda axial, determinando las relaciones que se pueden encontrar; y una última codificación selectiva, con el fin de analizar los datos de forma conjunta. Después de este análisis, han emergido un total de 14 códigos con los que se han establecido las distintas categorías para las citas.

\section{RESUlTADOS Y DisCUSIÓN}

Tal y como se puede observar a través de la Figura 1, se han diferenciado dichos códigos en función de la propuesta realizada a los sujetos, donde se les reclamó las distintas manifestaciones del bullying y las acciones que se podrían tomar para atajarlo. De ellos se puede extraer que hay una serie de códigos con mayor presencia que otros, es decir, tanto manifestaciones como acciones de mejora, o posibles soluciones, que están en el acervo colectivo de los individuos. De esta forma, las manifestaciones del bullying destacadas son el acoso físico y psicológico con un enraizamiento de 17 y 18 citas respectivamente. Por su lado, no existe una disparidad evidente entre las posibles soluciones que plantean los sujetos, únicamente se destaca la figura de autoridad, bien sean los padres, profesores o la policía, como elemento con mayor recurrencia en su mención.

Igualmente, cabe destacar que, tanto los códigos empleados para las manifestaciones como para las soluciones, han coincidido en mayor o menor medida con otros, creando vínculos entre ellos, apreciándose ello en la densidad mostrada en la Figura 1. 


\section{Figura 1}

Enraizamiento y densidad para los códigos empleados en el análisis

\begin{tabular}{|c|c|c|c|c|c|}
\hline & Nombre & Enraizamiento & & Densidad & \\
\hline$\bullet$ & $\checkmark$ Acecho/intimidación & 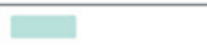 & 6 & 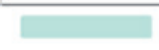 & 2 \\
\hline - & $\diamond$ Acoso ante la discapacidad & $\square$ & 4 & & 0 \\
\hline • & $\triangleright$ Acoso físico & 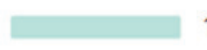 & 17 & 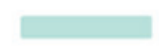 & 2 \\
\hline • & $\diamond$ Acoso psicológico & $\square$ & 18 & & 2 \\
\hline$\bullet$ & $>$ Acoso sexual & $\|$ & 1 & & 0 \\
\hline$\bullet$ & $\diamond$ Acoso verbal & 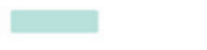 & 8 & $\mathbf{b}^{2}$ & 2 \\
\hline$\bullet$ & $\diamond$ Actividades de carácter educativo/formativo & a & 2 & $\square$ & 1 \\
\hline$\bullet$ & $\diamond$ Diálogo con el acosado & & 0 & & 0 \\
\hline$\bullet$ & $\diamond$ Diálogo con el acosador & $\square$ & 4 & & 3 \\
\hline$\bullet$ & $\diamond$ Diálogo con la familia & $\square$ & 5 & 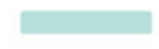 & 2 \\
\hline$\bullet$ & $\checkmark$ Diálogo entre acosado y acosador & $\|$ & 1 & & 0 \\
\hline$\bullet$ & $\diamond$ Diálogo entre amigos & 마 & 3 & & 0 \\
\hline$\bullet$ & $>$ Mediación & 1] & 2 & $\square$ & 1 \\
\hline$\bullet$ & $\diamond$ Recurrir a figura de autoridad & 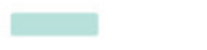 & 8 & 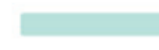 & 3 \\
\hline
\end{tabular}

Para un estudio con mayor profundidad de estas relaciones, se ha empleado la herramienta que facilita ATLAS.ti para el análisis de las co-ocurrencias entre códigos, tanto para las manifestaciones del bullying como para las soluciones, arrojando los coeficientes ${ }^{5}$ de co-ocurrencia que se muestran en las Tablas 1 y 2.

\section{Tabla 1}

Coeficientes entre códigos para las manifestaciones del bullying

\begin{tabular}{|c|c|c|c|c|c|c|c|}
\hline & 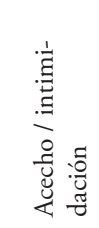 & 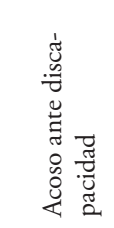 & $\begin{array}{l}\stackrel{8}{0} \\
i=0 \\
0 \\
0 \\
0 \\
\dot{0}\end{array}$ & 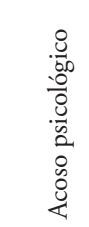 & 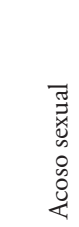 & $\begin{array}{l}\overline{0} \\
\overline{0} \\
\overline{0} \\
0 \\
0 \\
0 \\
0 \\
0\end{array}$ & 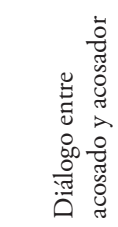 \\
\hline Acecho/intimidación & 0,00 & 0,00 & 0,10 & 0,28 & 0,00 & 0,00 & 0,00 \\
\hline Acoso ante la discapacidad & 0,00 & 0,00 & 0,11 & 0,24 & 0,00 & 0,00 & 0,00 \\
\hline Acoso físico & 0,10 & 0,11 & 0,00 & 0,21 & 0,00 & 0,14 & 0,00 \\
\hline Acoso psicológico & 0,28 & 0,24 & 0,21 & 0,00 & 0,06 & 0,19 & 0,00 \\
\hline Acoso sexual & 0,00 & 0,00 & 0,00 & 0,06 & 0,00 & 0,13 & 0,00 \\
\hline Acoso verbal & 0,00 & 0,00 & 0,14 & 0,19 & 0,13 & 0,00 & 0,00 \\
\hline Diálogo con la familia & 0,00 & 0,00 & 0,00 & 0,00 & 0,00 & 0,00 & 0,00 \\
\hline
\end{tabular}

\footnotetext{
${ }^{5}$ Los coeficientes de coocurrencia en ATLAS.ti indican la fuerza de relación entre dos códigos, siendo equiparable a un coeficiente de correlación.
} 
A través de la Tabla 1, las manifestaciones no muestran unos coeficientes de co-ocurrencia excesivamente altos, que sería lo apropiado para expresar vinculaciones fuertes entre esos códigos. Sin embargo, sí se halla una coincidencia que relaciona cuatro de ellos de forma significativa, las manifestaciones de \#Acoso psicológico, \#Acoso físico, \#Acoso ante la discapacidad y \#Acecho/intimidación. Pudiendo establece entre ellos, y a través de sus citas coincidentes, las relaciones que se muestran en la Figura 2.

\section{Figura 2}

Red semántica de relaciones entre manifestaciones del bullying

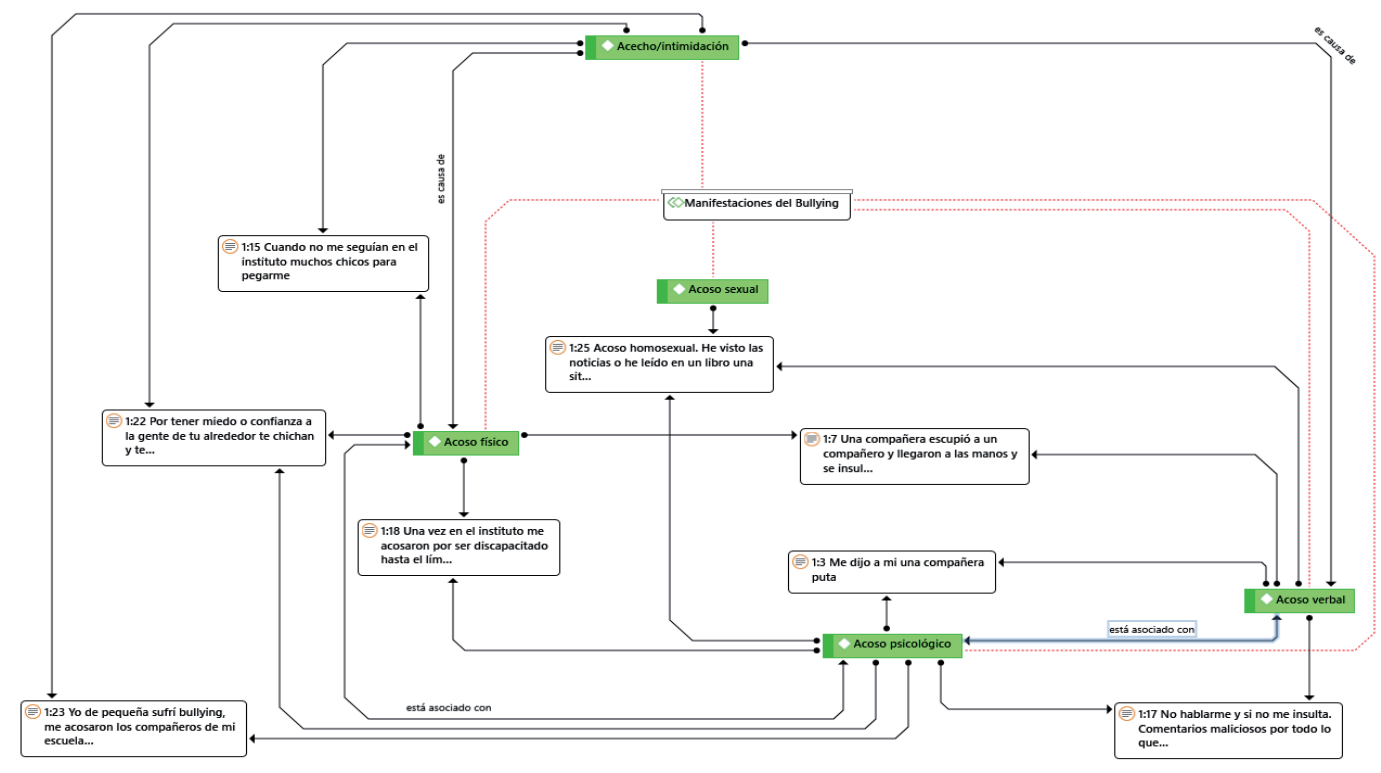

Como se puede apreciar, y así se indica anteriormente, el acoso psicológico y el acoso físico se tornan el centro de las distintas manifestaciones de esta problemática y, a su vez, asociados por la coincidencia de citas. Estas situaciones de acoso han terminado tejiendo una red para las personas con discapacidad intelectual, basada en críticas y percepciones negativas sobre la discapacidad intelectual (Mañas et al., 2020), que se va clonando y tomando forma en los centros educativos, hasta dar como resultado una identidad deteriorada (Barron, 2002). Así, este colectivo sufre en su piel cómo la "etiqueta que los diferencia” (Mañas et al., 2020, p.65) deriva en exclusión social (Goicoechea \& Bledma, 2017).

Es así como se va forjando esa tan poco conveniente predisposición social de las personas con discapacidad intelectual hacia el acoso escolar (Luengo Latorre \& Domínguez Gómez, 2015).

Una vez en el instituto me acosaron por ser discapacitado hasta el límite de que casi me quemaron el pelo con un mechero. (D1:18)

Por tener miedo o confianza a la gente de tu alrededor te chinchan y te golpean y me seguían, ser sincero y por eso te dicen que eres mala. Coger una chaqueta y ponérmela en la cabeza y darme como si fuera un tambor. (D1:22) 
Así mismo, estos códigos también se encuentran vinculados por otros códigos, como el acoso sexual, acoso verbal y el acecho/intimidación, tejiendo entre ellos, una red que muestro como una forma de manifestación, fácilmente, puede llevar a una de las otras. Aunque no existe una linealidad patente, si se observa como el acecho/intimidación del individuo es causa de un posterior acoso verbal que, a su vez, puede manifestarse como acoso físico y/o psicológico, además de poder ser, eventualmente, sexual. Todas estas conductas hostiles y agresivas "de diversa naturaleza: burlas, amenazas, intimidaciones, agresiones físicas, aislamiento sistemático o insultos" (Díaz-Aguado, 2005, p.549), procedentes de la falta de empatía y aceptación de las diferencias, a menudo derivan en acoso psicológico (Hernández et al., 2010). Son diversos los estudios que han demostrado que sufrir acoso o bullying (físico, psicológico o de exclusión social), provoca graves consecuencias negativas, como desajuste psicológico, ansiedad, depresión o incluso riesgo de suicidio (Klomek et al., 2007).

Cuando no me seguían en el instituto muchos chicos para pegarme. (D1:15)

Yo de pequeña sufrí bullying, me acosaron los compañeros de mi escuela e instituto, tenía siempre bullying y siempre fui al psicólogo. (D1:23)

No hablarme y si no me insulta. Comentarios maliciosos por todo lo que digo o hago. Por ser calla o hablar. Por dar consejos y esa persona cabrearse y contraatacar. Por saber otras palabras distintas a las de la otra persona. (D1:17)

La violencia verbal y la violencia psicológica, dentro del entorno educativo (Arellano, 2008), pone de manifiesto que la intencionalidad está dirigida a que la víctima pierda su autoestima, seguridad y capacidad para expresarse, reduciendo a la víctima al mínimo exponente.

Por otra parte, a la hora de plantear las distintas soluciones, ya se indicaba anteriormente a la figura de autoridad como código destacado ante esta situación, que tiene un nivel de co-ocurrencia considerable con las actividades de carácter formativo y el diálogo con la familia sobre el hecho ocurrido. Sin embargo, cuando se abordan las tablas de co-ocurrencias generada para las acciones planteadas por los sujetos, se distingue una relación que adquieren mayor vinculación que el resto, dado que alcanza un coeficiente de 0’50, esta es, el código asignado a actividades expresas de mediación y el referente a diálogo entre acosado y acosador.

Yo le ayudaría a esa persona para hablar con otra persona que había metido con la otra persona. (D2:7) 
Tabla 2

Coeficientes entre códigos para las soluciones del bullying

\begin{tabular}{|c|c|c|c|c|c|c|c|}
\hline & 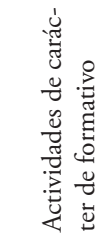 & 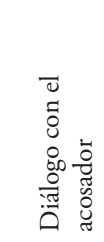 & 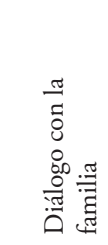 & 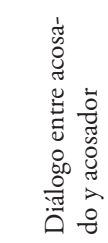 & 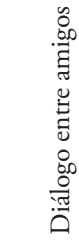 & 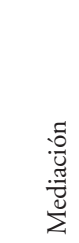 & 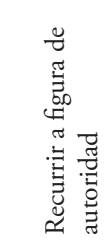 \\
\hline $\begin{array}{l}\text { Actividades de carácter } \\
\text { formativo }\end{array}$ & 0,00 & 0,20 & 0,00 & 0,00 & 0,00 & 0,00 & 0,25 \\
\hline Diálogo con el acosador & 0,20 & 0,00 & 0,13 & 0,00 & 0,00 & 0,20 & 0,09 \\
\hline Diálogo con la familia & 0,00 & 0,13 & 0,00 & 0,00 & 0,14 & 0,00 & 0,18 \\
\hline $\begin{array}{l}\text { Diálogo entre acosado y } \\
\text { acosador }\end{array}$ & 0,00 & 0,00 & 0,00 & 0,00 & 0,00 & 0,50 & 0,00 \\
\hline Diálogo entre amigos & 0,00 & 0,00 & 0,14 & 0,00 & 0,00 & 0,00 & 0,10 \\
\hline Mediación & 0,00 & 0,20 & 0,00 & 0,50 & 0,00 & 0,00 & 0,00 \\
\hline Recurrir a figura de autoridad & 0,25 & 0,09 & 0,18 & 0,00 & 0,10 & 0,00 & 0,00 \\
\hline
\end{tabular}

Igualmente, se puede ver las relaciones entre códigos señaladas como co-ocurrentes y otras que, aunque no muestran relevancia en este aspecto, sí poseen significación a nivel semántico, establecidas entre los códigos señalados mediante la Figura 3.

\section{Figura 3}

Red semántica de relaciones entre soluciones al bullying

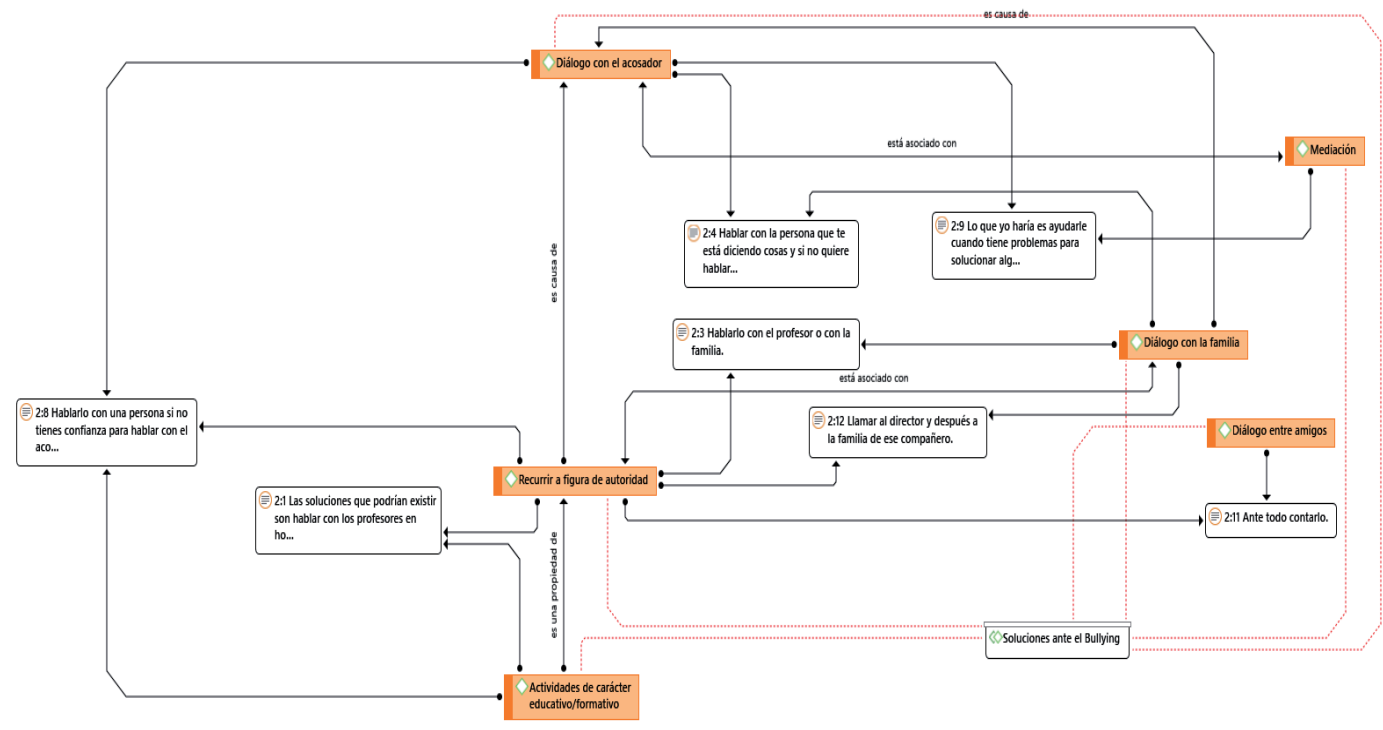


El bullying, al implicar conductas repetitivas de agresión, intimidación o aislamiento intencional contra un compañero que es incapaz de defenderse, genera un desequilibrio de poder (Olweus, 2013). Además, dicha opresión, sustentada sobre tradiciones, creencias e ideales que aun hoy día proporcionan una visión acotada de la discapacidad, ha conllevado, con el paso de los años, a que la discapacidad sea asumida como "un factor condicionante o de vulnerabilidad a la exclusión social del individuo" (Díaz, 2010, p. 128). Así, a través de la mencionada Figura 3 se refuerzan algunos de los aspectos destacados anteriormente, situando la imagen de autoridad como eje vertebral de las acciones contra el bullying, siendo el nexo entre el diálogo con la familia y el diálogo con el acosador. Lo que conlleva, de forma implícita, asociada la importante carga sobre la mediación que apuntan los sujetos en su exposición de soluciones. En este sentido cabe destacar la relevancia del rol del observador, sobre todo en momentos cruciales de la vida, como pueden ser la adolescencia o juventud, donde el estatus en el grupo de iguales es más importante (Salmivalli, 2014).

Actualmente, la red de iguales resulta esencial para romper la ley del silencio e identificar problemáticas, mejorando así la convivencia escolar y prevenir la violencia (Ortega et al., 2016). La omisión de socorro por parte de los compañeros y las conductas pasivas en situaciones en las que han sido observadores, pasivos o neutrales (Cerezo, 2001) generan que la situación se prolongase en el tiempo (Mañas et al., 2020). Es por ello que, en situación de vulnerabilidad, se recurra a roles jerárquicos más altos.

Hablar con la persona que te está diciendo cosas y si no quiere hablar, hablar con tus padres. (D2:4)

Hablarlo con una persona si no tienes confianza para hablar con el acosador. Hablar con el director y con los padres. Hacer cursos de cambio de papeles para prevenir el acoso. (D2:8)

\section{Conclusiones}

La existencia de fenómenos como el acoso y la victimización entre iguales, tan problemáticos y con tan altas tasas de prevalencia, está siendo objeto de estudio desde hace décadas, principalmente por los perjuicios causados a todos los implicados a nivel académico, social y personal (Hellfeldt et al., 2018; Salmivalli, 2014), siendo además reconocida a nivel mundial en todas las instituciones educativas (Zych et al., 2015). Por ello, cabe destacar la relevancia del estudio, abalada por la férrea voluntad de avanzar en la inclusión social de las personas con discapacidad intelectual (Pallisera et al., 2018).

Las instituciones, desgraciadamente, se han consagrado como un entorno de segregación y exclusión para colectivos vulnerables, como el de las personas con discapacidad intelectual (Connor \& Ferri, 2007; Mañas et al., 2020). Así, la experiencia educativa sigue siendo más que otra cosa, un ejercicio de supervivencia (Flores et al., 2010), que exige al alumnado que se adapte a ella. Por esto es tan relevante la formación y preparación docente, dada su demostrada influencia (Lalvani, 2015), ya que el docente con experiencia en trato con estudiantes con discapacidad es más receptivo y cercano que otro profesorado (Johnson, 2006).

El bullying, como violencia interpersonal entre iguales, se manifiesta en los contextos educativos a través de maltrato físico, psicológico o relacional. Este agravio se caracteriza por la intencionalidad del agresor, el desequilibrio de poder generado con respecto a la víctima o la frecuencia y duración en el que se desarrolla (Zych et al., 2015). 
Los procesos de exclusión, apoyados en el ideal de discapacidad como factor de inferioridad e incapacidad (Connor \& Ferri, 2007) mantienen a este colectivo en el silencio. Partiendo del principio de integración sustentado en la normalización de la vida de los alumnos con necesidades educativas especiales (Barrio de la Puente, 2009), el Programa Todos Somos Campus supone una apuesta decidida por la inclusión de las personas con discapacidad intelectual en la Universidad, persiguiendo convertir las voces de silencio, en voces de cambio (Seale, 2017). Con este fin, se esboza la realización de un proyecto formativo, capaz de dar respuesta a las necesidades individuales y de inclusión, para que los jóvenes con discapacidad intelectual puedan participar como miembros de pleno derecho en su comunidad educativa.

De esta forma, y a la luz de los resultados obtenidos, el desarrollo de estrategias positivas para resolver conflictos entre iguales de forma dialogada y la presión de la norma social de grupo correlacionan positivamente con la ayuda activa hacia las víctimas y negativamente con actuar con pasividad (Martín \& Casas, 2019). Además, se constata, al igual que ocurre en el trabajo de Pozzoli y Gini (2013), que estrategias negativas de resolución de conflictos correlacionan positivamente con la pasividad de los observadores. Cabe contemplar que las relaciones establecidas entre las manifestaciones del bullying y sus posibles soluciones no se presentan de forma aislada, sino que establecen vínculos que hacen de ellas una red de presencia para atajar esta problemática educativa.

Así, se pueden realizar una serie de aseveraciones teniendo en cuenta lo aquí expuesto: a) dentro de las manifestaciones del bullying existe un crecimiento progresivo de dichas acciones o se exteriorizan de forma simultánea; b) se vinculan de forma significativa diferentes hechos de violencia con la discapacidad o vulnerabilidad de los sujetos; c) se expresa la mediación y el dialogo como principales elementos de acción contra el bullying antes de llegar a reclamar la acción de una figura de autoridad, lo que implica el alto nivel emocional presente dentro de esta problemática.

Obviamente, las afirmaciones realizadas han de ser tomadas con cautela, ya que se trata de un estudio exploratorio con un número limitado de participantes, siendo ésta una de las limitaciones del presente trabajo y fuente de investigaciones futuras. Es por ello que se patenta la necesidad de trabajar en diversos frentes para abordar la cuestión que se ha desarrollado en el presente trabajo. Poniéndose el foco en la toma de medidas y distintas acciones que se demanda con urgencia y su avance o implementación no se adecua a tal demanda.

\section{REFERENCIAS}

Adams, F. D., \& Lawrence, G. J. (2011). Bullying victims: The effects last into college. American Secondary Education, 40(1), 4-11.

Alarcón, A., Munera, L., \& Montes, A. (2017). La teoría fundamentada en el marco de la investigación educativa. Saber, ciencia y libertad, 12(1), 236-245.

Arellano, N. (2008). Violencia entre pares escolares (bullying) y su abordaje a través de la mediación escolar y los sistemas de convivencia. Informe de Investigaciones Educativas, 22(2), 211-230.

Arnaiz, P. (2019). La educación inclusiva en el siglo XXI. Avances y desafíos. Murcia: Universidad de Murcia. 
Barrio de la Puente, J. L. (2009). Hacia una educación inclusiva para todos. Revista complutense de educación, 20(1), 13-31.

Barron, K. (2002). Who Am I? Women with Learning Difficulties (Re)constructing their SelfIdentity. Scandinavian Journal of Disability Research, 4(1), 58-79.

Belmonte, M. L., \& García-Sanz, M. P. (2013). La escuela de vida, otra mirada a la discapacidad intelectual. Revista Fuentes, 14, 147-170.

Bisquerra, R. (2014). Metodología de la Investigación educativa. La Muralla.

Cerezo, F. (2001). La violencia en las aulas. Análisis y propuestas de intervención. Pirámide.

Cerrillo, R., Izuzquiza, D., \& Egido, I. (2013). Inclusión de jóvenes con discapacidad intelectual en la Universidad. Revista de Investigación en Educación, 11(1), 41-57.

Connor, D. J., \& Ferri, B. A. (2007). The conflict within: resistance to inclusion and other paradoxes in special education. Disability and Society, 22(1), 63-77.

Díaz, E. (2010). Ciudadanía, identidad y exclusión social de las personas con discapacidad. Política y Sociedad, 47(1), 115-135.

Díaz-Aguado, M. J. (2005). La violencia entre iguales en la adolescencia y su prevención desde la escuela. Psicothema, 17(4), 549-558.

Díez, A. M., López, R. G., \& Molina, V. M. (2015). Students with Disabilities in Higher Education: a Biographical-Narrative Approach to the Role of Lecturers. Higher Education Research and Development, 34, 147-159. https://doi.org/10.1080/07294360.2014.934329

Flores, J. I. R., Mendéz, A. E. L., González, P. C., García, M. J. M., \& Arcos, D. P. (2010). La configuración de identidades en la experiencia escolar. Escenarios, sujetos y regulaciones. Revista de Educación, 353, 187-209.

Gairín, J., \& Suárez, C. (2014). Clarificar e identificar los grupos vulnerables. En J. Gairín (coord.). Colectivos vulnerables en la universidad: reflexiones y propuestas para la intervención (pp. 33-61). Wolters Kluwer.

Gálvez, J. A. (2012). Análisis empírico del consumo, saliencia y credibilidad mediática como factores explicativos del posicionamiento actitudinal hacia la inmigración. Empiria, 24, 67-92. https://doi. org/10.5944/empiria.24.2012.843

García-Sanz, M. P. (2012). Fundamentos teóricos y metodológicos de la evaluación de programas. Murcia: Diego Marín.

Goicoechea, P. H., \& Bledma, F. J. R. (2017). Las familias ante el acoso escolar. Revista de Estudios de Juventud,115, 139-152.

González Luna, B. (2013). Investigando con personas con dificultades de aprendizaje. Revista Española de Discapacidad, 1(2), 77-94.

Hardy, I. \& Woodcock, S. (2015). Inclusive education policies: discourses of difference, diversity and deficit. International Journal of Inclusive Education, 19(2), 141-164.

Heikkilä, M., Katsui, H., \& Mustaniemi-Laakso, M. (2020). Disability and vulnerability: a human right reading of the responsive state. The International Journal of Human Rights, 1-21. https://doi.org/1 $0.1080 / 13642987.2020 .1715948$ 
Hellfeldt, K., Gill, P. E., \& Johansson, B. (2018). Longitudinal analysis of links between bullying victimization and psychosomatic maladjustment in Swedish schoolchildren. Journal of School Violence, 17(1), 86-98. https://doi.org/10.1080/15388220.2016.1222498

Hernández, S., Fernández, C., \& Baptista, L. (2010). Metodología de la investigación. McGraw-Hill.

Houchins, D. E., Oakes, W. P. \& Johnson, Z. G. (2016). Bullying and students with disabilities: A systematic literature review of intervention studies. Remedial and Special Education, 37(5), 259273. https://doi.org/10.1177\%2F0741932516648678

Jahoda, A., \& Markova, I. (2004). Coping with social stigma: People with intellectual disabilities moving from institutions and family home. Journal of Intellectual Disability Research, 48(Pt 8), 719-729. https://doi.org/10.1111/j.1365-2788.2003.00561.x

Johnson, A. L. (2006). Students with disabilities in postsecondary education: Barriers to success and implication to professionals. Vistas online http://counselingoutfitters.com/vistas2006

Klomek, A. B., Marrocco, F., Kleinman, M., Schonfeld, I. S. \& Gould, M. S. (2007). Bullying, depression, and suicidality in adolescents. Journal of the American Academy of Child y Adolescent Psychiatry, 46(1), 40-49.

Lalvani, P. (2015). Disability, stigma and otherness: Perspectives of parents and teachers. International Journal of Disability, Development and Education, 62(4), 379-393. https://doi.org/10.1080/1034 912X.2015.1029877

Lipka, O., Baruch, A. F., \& Meer, Y. (2019). Academic support model for post-secondary school students with learning disabilities: student and instructor perceptions. International Journal of Inclusive Education, 23(2), 142-157. https://doi.org/10.1080/13603116.2018.1427151

Luengo Latorre, A. J., \& Domínguez Gómez, I. M. (2015). Discapacidad, Infancia y Acoso. En J. M. Fernández Martínez, \& C. Ganzenmüller (Dir.). Discapacidad e Infancia (pp. 199-247). Consejo General del Poder Judicial.

Mañas, M., González, B., \& Cortés, P. (2020). Historias de vida de personas con discapacidad intelectual: Entre el acoso y exclusión en la escuela como moduladores de la identidad. Revista Educación, Politica y Sociedad, 5(1), 60-84.

Martín, J. M., \& Casas, J. A. (2019). Evaluación del efecto del programa "Ayuda entre iguales de Córdoba” sobre el fomento de la competencia social y la reducción del Bullying. Aula abierta, 48(2), 221-228.

McMillan, J. \& Schumacher, S. (2005). Investigación educativa. PEARSON.

Melero, N., Moriña, A., \& Perera, V. H. (2019). Acciones del profesorado para una práctica inclusiva en la universidad. Revista Brasileira de Educação, 24, 1-19. http://doi.org/10.1590/s141324782019240016

Miles, M. B., Huberman, A. M., \& Saldaña, J. (2014). Qualitative Data Analysis. A Methods Sourcebook (3a Ed.). Sage.

Moriña, A. (2017). Investigar con historias de vida. Metodología biográfico-narrativa. Narcea.

Newman, G. D. (2006). El razonamiento inductivo y deductivo dentro del proceso investigativo en ciencias experimentales y sociales. Laurus, 12(extra), 180-205.

Olweus, D. (2013). School bullying: Development and some important challenges. Annual Review of Clinical Psychology, 9, 751-780. https://doi.org/10.1146/annurev-clinpsy-050212-185516 
Onghena, P., Maes, B., \& Heyvaert, M. (2019). Mixed methods single case research: State of the art and future directions. Journal of Mixed Methods Research, 13(4), 461-480.

Ortega, R., Del Rey, R., \& Casas, J. A. (2016). La convivencia escolar: Clave en la predicción del bullying. Revista Iberoamericana De Evaluación Educativa, 6(2), 91-102.

Pallisera, M., Fullana, J., Puyaltó, C., Vilá, M., Díaz, G., \& Castro, M. (2018). Retos para la vida independiente de las personas con discapacidad intelectual. Revista Española de Discapacidad, 6(1), 7-29.

Pereira, Z. (2011). Los diseños de método mixto en la investigación en educación: una experiencia concreta. Revista Electrónica Educare, 15(1), 15-29.

Pozzoli, T. \& Gini, G. (2013). Why do bystanders of bullying help or not? A multidimensional model. The Journal of Early Adolescence, 33(3), 315-340.

Riddell, S., \& Weedon, E. (2014). Disabled students in higher education: Discourses of disability and the negotiation of identity. International Journal of Educational Research, 63, 38-46. https://doi. org/0.1016/j.ijer.2013.02.008

Rose, C. A., Monda-Amaya, L. E., \& Espelage, D. L. (2011). Bullying perpetration and victimization in special education: A review of the literature. Remedial and Special Education, 32(2), 114-130. https://doi.org/10.1177\%2F0741932510361247

Salmivalli, C. (2014). Participant roles in bullying: How can peer bystanders be utilized in interventions? Theory into Practice, 53(4), 286-292.

San Martín, D. (2014). Teoría fundamentada y Atlas. ti: recursos metodológicos para la investigación educativa. Revista electrónica de investigación educativa, 16(1), 104-122.

Seale, J. (2017). From the voice of a "socratic gadfly": A call for more academic activism in the researching of disability in postsecondary education. European Journal of Special Needs Education, 32, 153-169. https://doi.org/10.1080/08856257.2016.1254967

Strand, M., Benzein, E., \& Saveman, B. I. (2004). Violence in the care of adult persons with intellectual disabilities. Journal of clinical nursing, 13(4), 506-514.

Strauss, A., \& Corbin, J. (1998). Basics of qualitative research: Techniques and procedures for developing grounded theory (2nd ed.). Sage.

Suddaby, R. (2006). From the editors: What grounded theory is not. The Academy of Management Journal, 49(4), 633-642.

Thomas, L. (2016). Developing inclusive learning to improve the engagement, be- longing, retention, and success of students from diverse groups. In M. Shah, A. Bennett, \& E. Southgate (Eds.), Widening higher education participation. A global perspective (pp. 135-159). Elsevier.

Tipton-Fisler, L. A., Rodríguez, G., Zeedyk, S. M., \& Blacher, J. (2018). Stability of bullying and internalizing problems among adolescents with ASD, ID, or typical development. Research in Developmental Disabilities, 80, 131-141. https://doi.org/10.1016/j.ridd.2018.06.004

Veitch, S., Strehlow, K., \& Boyd, J. (2018). Supporting university students with socially challenging behaviours through profesional development for teaching staff. Journal of Academic Language \& Learning, 12(1), A156-A167.

Vessey, J. A., \& O’Neill, K. M. (2011). Helping students with disabilities better address teasing and bullying situations: A MASNRN study. The Journal of School Nursing, 27(2), 139-148. https://doi. org/10.1177\%2F1059840510386490 
Wei, H. S., Chang, H. H., \& Chen, J. K. (2016). Bullying and victimisation among Taiwanese students in special schools. International Journal of Disability, Development and Education, 63(2), 246-259. https://doi.org/10.1080/1034912X.2015.1092505

Zeedyk, S. M., Rodriguez, G., Tipton, L. A., Baker, B. L. \& Blacher, J. (2014). Bullying of youth with autism spectrum disorder, intellectual disability, or typical development: Victim and parent perspectives. Research in Autism Spectrum Disorders, 8(9), 1173-1183. https://doi.org/10.1016/j. rasd.2014.06.001

Zych, I., Ortega, R., \& Del Rey, R. (2015). Systematic review of theoretical studies on bullying and cyberbullying: Facts, knowledge, prevention, and intervention. Aggression and Violent Behavior, 23, 1-21. https://doi.org/10.1016/j.avb.2015.10.001-

Recebido em: 19/02/2020

Reformulado em: 01/04/2020

Aprovado em: 07/04/2020 
ALMAGRO, M.L.B.; GÓMEZ, A.B.; RUIZ, A.B.M. 